379 A COMPARISON OF PATIENTS' AND PHYSICIANS' ASSESSMENTS OF DISEASE ACTIVITY USING THE SWEDISH VERSION OF THE SYSTEMIC LUPUS ACTIVITY QUESTIONNAIRE

${ }^{1} S$ Pettersson*, ${ }^{2} E$ Svengsson, ${ }^{2} \mathrm{~J}$ Gustafsson, ${ }^{1} S$ Möller, ${ }^{2}$ I Gunnarsson, ${ }^{3}$ E Welin Henriksson. ${ }^{1}$ Karolinska University Hospital, Rheumatology Unit- D201, Stockholm, Sweden; ${ }^{2}$ Karolinska Institutet, Rheumatology Unit- Department of Medicine- Solna, Stockholm, Sweden; ${ }^{3}$ Linköpings Universitet, Division of Nursing Science- Department of Medical and Health Sciences-, Linköping, Sweden

\subsection{6/lupus-2017-000215.379}

Background and aims We compared patients' assessments of Systemic Lupus Erythematosus (SLE) disease activity by a Swedish version of the Systemic Lupus Activity Questionnaire
(SLAQ) questionnaire with physicians' assessments by Systemic Lupus Activity Measure (SLAM) and SLE Disease Activity Index (SLEDAI). Additionally, we explored the performance of the SLAQ in patients with short $(<1$ year) versus long $(\geq 1$ year) disease duration.

Methods Patients filled out SLAQ prior to physicians' assessments. Correlations between SLAQ-total, sub-scales (Symptom score, Flares, Patients global) and SLAM and SLEDAI as well as between the corresponding items in SLAQ and SLAM were evaluated using Spearman's $\rho$. Comparisons between patients with different disease durations were performed with MannWhitney U or Chi-square tests.

Results We included 203 patients (79\% women, median age 45 (IQR 33-57) years, disease duration 5 (IQR 0-14) years)

Abstract 379 Table 1 Characteristics of the 203 participants with SLE (women 79\%).

\begin{tabular}{|c|c|c|c|c|}
\hline & Median & IQR & Minimum & Maximum \\
\hline Age (years) & 45 & $33-57$ & 18 & 82 \\
\hline Disease duration (years) & 5 & $0-14$ & 0 & 58 \\
\hline Symptom score $(0-24)$ & 10 & $6-14$ & 0 & 24 \\
\hline SLAQ* score $(0-47)$ & 12 & $6-18$ & 0 & 40 \\
\hline Flare $^{b}(0-3)$ & 1 & $0-2$ & 0 & 2 \\
\hline $\operatorname{PNRS}^{\circ}(0-10)$ & 4 & $1-7$ & 0 & 10 \\
\hline SLAM d & 6 & $3-10$ & 0 & 24 \\
\hline SLAM nolab & 5 & $2-7$ & 0 & 21 \\
\hline SLEDAI ${ }^{*}$ & 4 & 0.7 & 0 & 26 \\
\hline SLICC/ACR DI ${ }^{f}$ & 1 & $0-2$ & 0 & 10 \\
\hline
\end{tabular}

-SLAQ = Systemic Lupus Activity Questionnaire

b Flare: patient global assessment of severity of lupus flare, Missing=32

-PNRS: patient numerical rating scale of global disease activity. Missing=11

¿SLAM = Systemic Lupus Activity Measure

- SLEDAI = SLE Disease Activity Index, Missing =1

${ }^{\mathrm{S}} \mathrm{LICC} / \mathrm{ACR}$ DI $=$ Systemic Lupus International Collaboration Clinics/American College of Rheumatology damage index 
(Table 1). Correlations between physicians' SLAM, without laboratory items (SLAM-nolab), and patients' assessments were: SLAQ total, $\rho=0.685$, Symptom score, $\rho=0.651$, Flares, $\rho=0.547$ and Patients global, $\rho=0.600$. Of symptom items fatigue $(\rho=0.640)$, seizures $(\rho=0.635)$ and headache $(\rho=0.604)$ correlated best. Neurology/stroke syndrome, skin and lymphadenopathy correlated less well $(\rho<0.24)$. Patients' and physicians' assessments were notably more discordant for patients with short disease durations.

Conclusions We confirm that the SLAQ can be used to monitor disease activity. However, the discrepancy between patients' and physicians' assessments was greater for patients with short versus long disease duration. We encourage further use of the SLAQ, but would like to develop a shorter version which would be valuable in modern, partly web-based, clinical care.

\section{COMPARISONS OF FATIGUE, ANXIETY AND DEPRESSION IN SYSTEMIC LUPUS ERYTHEMATOSUS, SYSTEMIC SCLEROSIS AND ANTI-NEUTROPHIL CYTOPLASMATIC ANTIBODY-ASSOCIATED VASCULITIS}

${ }^{1,2}$ S Pettersson* ${ }^{3}$ A Nordin, ${ }^{3}$ E Svenungsson, ${ }^{3}$ I Gunnarsson. ${ }^{1}$ Karolinska University Hospital, Rheumatology Unit- D201, Stockholm, Sweden; ${ }^{2}$ Karolinska Institutet, Division of Physiotherapy- Department of Neurobiology- Care Sciences and Society, Stockholm, Sweden; ${ }^{3}$ Karolinska Institutet- Karolinska University Hospital, Department of Medicine- Unit of Rheumatology- Karolinska University Hospital-, Stockholm, Sweden

\subsection{6/lupus-2017-000215.380}

Background and aims To compare fatigue, anxiety and depression between systemic lupus erythematosus (SLE), systemic sclerosis (SSc) and anti-neutrophil cytoplasmatic antibody associated vasculitis (AAV).

Methods Patients filled in self-assessments of fatigue (Multi Assessment of Fatigue Scale, MAF) and anxiety/depression

Abstract 380 Table 1 Comparision of fatigue between three cohorts ${ }^{\mathrm{a}}$ of inflamatory disorder.

\begin{tabular}{|c|c|c|c|c|c|c|}
\hline & SLE & SSc & AAV & & p-value $e^{b}$ & \\
\hline & Median (IQR) & Median (IQR) & Median (IQR) & $\begin{array}{c}\text { SLE } \\
\text { vs SSc }\end{array}$ & $\begin{array}{c}\text { SLE } \\
\text { vS } \\
\text { AAV. }\end{array}$ & $\begin{array}{l}\text { SSc vs } \\
\text { AAV. }\end{array}$ \\
\hline Age (years) & $47(34-57)$ & $60(52-69)$ & $62(49-69)$ & $<0.001$ & $<0.001$ & 0.296 \\
\hline Disease (years) & $10(2-20)$ & $2(0-8)$ & $3(0-8)$ & $<0,001$ & $<0.001$ & 0.807 \\
\hline \multicolumn{7}{|l|}{ Fatigue } \\
\hline Global' $(1-50)$ & $30.4(21.4-38.9)$ & $26.9(18.5-35.1)$ & $27.8(18.7-33.7)$ & 0.001 & 0.001 & 0.899 \\
\hline $\operatorname{Degree}^{c}(1-10)$ & $7(5-9)$ & $6(4-8)$ & $7(4-8)$ & $<0.001$ & 0.002 & 0.429 \\
\hline Severity $^{c}(1-10)$ & $6(4-8)$ & $6(4-7)$ & $6(3-7)$ & 0.001 & 0.002 & 0.803 \\
\hline Distress $^{d}(1-10)$ & $6(3-8)$ & $5(2-7)$ & $5(3-7)$ & 0.007 & 0.005 & 0.956 \\
\hline Activities $^{\text {d }}(1-10)$ & $3.6(1.8-5.6)$ & $3.4(1.8-4.9)$ & $3.0(1.9-4.4)$ & 0.084 & 0.010 & 0.358 \\
\hline Anxiety $^{\circ}(0-14)$ & $6(3-9)$ & $5(2-7.8)$ & $5(2-9)$ & 0.013 & 0.007 & 0.767 \\
\hline Depression $^{\mathrm{C}}(0-14)$ & $4(2-7)$ & $4(2-6.8)$ & $4(1-7)$ & 0.596 & 0.191 & 0.453 \\
\hline \multicolumn{7}{|c|}{$\begin{array}{l}\text { a:SLE=systemic iupus erythematosus, } S S C=\text { systemic sclerosis, } A A V=\text { and anti-neutrophil cytoplasmatic antibody } \\
\text { associated vasculitis }\end{array}$} \\
\hline \multicolumn{7}{|c|}{ b:Mann_Whitmey $U$} \\
\hline \multicolumn{7}{|c|}{ c: Fatigue components from Multi Assessment of Fatigue Scale } \\
\hline d: Hospital Anxiet & nd Depression Scale. & & & & & \\
\hline
\end{tabular}

